\title{
La lithotritie biliaire extracorporelle
}

L'équipe de Munich avait rapporté en 1986 ses premiers résultats de la lithotritie extracorporelle par ondes de chocs électrohydrauliques dans la lithiase vésiculaire, portant sur neuf malades [1]. Les auteurs ont maintenant étendu leurs observations sur 175 malades ayant une lithiase vésiculaire symptomatique[2]. Ces malades ont été sélectionnés à partir de 2010 patients adressés en vue du traitement, parmi lesquels 565 (28\%) ont été sélectionnés pour la lithotritie, et, à ce jour, 175 traités. Les critères de sélection ont été : calculs radiotransparents de moins de trois $\mathrm{cm}$, moins de trois calculs, vésicule opacifiée par cholécystographie orale, antécédents de colique hépatique (donc lithiase symptomatique). Les raisons d'exclusion ont été des calculs trop nombreux dans $44 \%$ des cas, des calculs calcifiés dans $15 \%$ des cas, des calculs de plus de trois $\mathrm{cm}$ dans $8 \%$ des cas, des calculs asymptomatiques dans $6 \%$ des cas. Il y avait $125 \mathrm{femmes}$ et 50 hommes, âgés de 17 à 77 ans. Cent quarante-cinq patients avaient un calcul unique et 30 patients avaient deux ou trois calculs. L'appareil est à ondes de choc électrohydrauliques (Dornier) et le repérage des calculs se fait par échographie. En moyenne, 1200 ondes de choc ont été délivrées en 13 à 165 minutes (médiane 41 minutes). Les 54 premiers patients ont été traités sous anesthésie générale, les suivants ayant reçu des opiacés par voie intraveineuse, une anesthésie épidurale ou même pas d'anesthésie du tout. Neuf patients ont eu deux séances. Tous les patients ont reçu un traitement médical par l'association acide chénodésoxycholique et acide ursodésoxycholique $(7,5 \mathrm{mg} / \mathrm{kg}$ / jour de chaque) jusqu'à trois mois après la disparition complète des calculs.

Une fragmentation a été obtenue chez tous les malades sauf un. Les calculs ont complètement disparu chez $30 \%$ des patients après deux mois, $48 \%$ en deux à quatre mois, $63 \%$ en quatre à huit mois, $78 \%$ en huit à douze mois, et $91 \%$ en 12 à 18 mois. Le pourcentage de disparition complète était plus élevé chez les malades ayant un calcul unique de moins de $20 \mathrm{~mm}$, étant dans ce cas de $95 \%$ à 18 mois.

La taille des calculs paraît être un déterminant important du succès final. Par exemple, à huit mois, les calculs avaient complètement disparu chez $78 \%$ des malades ayant un calcul de moins de $20 \mathrm{~mm}$, contre $51 \%$ seulement chez ceux ayant un calcul de 21 à $30 \mathrm{~mm}(\mathrm{p}<0,01)$. Les fragments obtenus sont d'ailleurs plus petits dans le premier cas (2,2 $\mathrm{mm}$ pour le plus gros) que dans le second $(2,7 \mathrm{~mm} ; \mathrm{p}<0,01)$. Le nombre est aussi important, puisqu'au même temps (huit mois), seulement $29 \%$ des malades ayant des calculs multiples avaient vu ceux-ci complètement disparaître. Les malades ayant des calculs multiples avaient d'ailleurs, en moyenne, des fragments plus gros $(4,6 \mathrm{~mm})$ que ceux ayant des calculs uniques $(2,4 \mathrm{~mm} ; \mathrm{p}<0,001)$.

Les effets secondaires ont été relativement peu fréquents. Des pétéchies cutanées ont été observées chez $14 \%$ des patients ; elles ont été bénignes et réversibles. Une hématurie macroscopique a été notée chez $3 \%$ des patients, probablement du fait de microtraumatismes du rein droit traversé par les ondes de choc. Des coliques hépatiques ont été observées chez $30 \%$ des patients : étant donné que tous les patients avaient des coliques hépatiques avant la lithotritie, il est difficile de dire combien de ces coliques hépatiques pourraient être liées à la fragmentation elle-même. Enfin, deux malades ont eu une pancréatite qualifiée de modérée et une sphinctérotomie endoscopique a été effectuée chez l'un de ces malades. Un malade ayant des coliques hépatiques et une fragmentation insuffisante a eu une cholécystectomie.

Cette étude bien conduite confirme les résultats de l'étude préliminaire. Elle montre que la lithotritie électrohydraulique extracorporelle, associée au traitement par les acides biliaires, est un traitement efficace et sans risque de la lithiase vésiculaire dans des cas bien sélectionnés. Certains problèmes restent bien sûr à résoudre : quel est le pourcentage de récidives et quelle attitude faut-il adopter après la disparition complète des calculs? Quelle est l'efficacité sur les calculs calcifiés? (Le traitement a été tenté chez 25 patients ayant des calculs calcifiés, avec 24 fragmentations et une disparition de calculs chez trois malades sur dix suivis pendant six mois). Quelle est l'efficacité des autres lithotriteurs, à ultrasons notamment? Finalement, quelle est la proportion de l'ensemble des malades lithiasiques à qui le traitement peut s'adresser? Dans l'étude, $28 \%$ des malades adressés en vue du traitement ont pu être traités ou vont l'être, mais le biais de sélection est évident. D'autres études devront répondre à ces questions, mais d'ores et déjà le travail de l'équipe munichoise a ouvert la voie à une nouvelle et passionnante possibilité thérapeutique de la lithiase vésiculaire.

S.E.

1. Sauerbruch T, Delius M, Paumgartner G, et al. Fragmentation of gallstones by extracorporeal shock waves. $N$ Engl J Med 1986; 314 : $818-22$.

2. Sackmann M, Delius M, Sauerbruch T, et al. Shock-wave lithotrypsy of gallbladder stones. The first 175 patients. N Engl J Med 1988 ; 318: 393-7. 